\title{
EFSUMB Young Investigator Winners 2021
}

\section{Joint first prize for best \\ technical presentation}

Improved drug delivery to the brain with Rapid Short-Pulses of focused ultrasound and microbubbles

Sophie V Morse ${ }^{1}$, Tiffany G Chan, Nicholas ] Long $^{2}$, James J Choi ${ }^{1}$

${ }^{1}$ Department of Bioengineering, Imperial College London, London, SW7 2BP, UK

${ }^{2}$ Department of Chemistry, Imperial College London, Molecular Sciences Research Hub, London, W12 0BZ, UK

\section{ABSTRACT}

\section{Introduction}

Focused ultrasound and microbubbles can locally and noninvasively open the blood-brain barrier, allowing drugs into the brain. Clinical results in adults have been encouraging, however, there are concerns for more sensitive patients - elderly with dementia and children with brain cancer. With current ultrasound methods, drugs are delivered inefficiently to diseased regions with side effects including unpredictable drug distributions, the en- trance of red blood cells and neurotoxic proteins, and hour-long tissue recovery.

\section{Methods}

We have developed a Rapid Short-Pulse (RaSP) ultrasound sequence designed to improve drug delivery. We have evaluated the ability of RaSP to improve the performance and safety of drug delivery, by investigating the drug dose, distribution, tissue damage, immune response and tissue recovery in comparison with conventional long-pulses.

\section{Results}

Despite depositing 150 times less energy into the brain, RaSP sequences delivered drugs more uniformly with a similar dose to long-pulses. RaSP resulted in the barrier closing within just 10 minutes rather than hours, with less neurotoxic proteins entering the brain. The immune response with RaSP was also reduced and no red blood cell extravasation or damage was observed.

\section{Conclusion}

These results indicate that RaSP sequences will significantly improve the performance

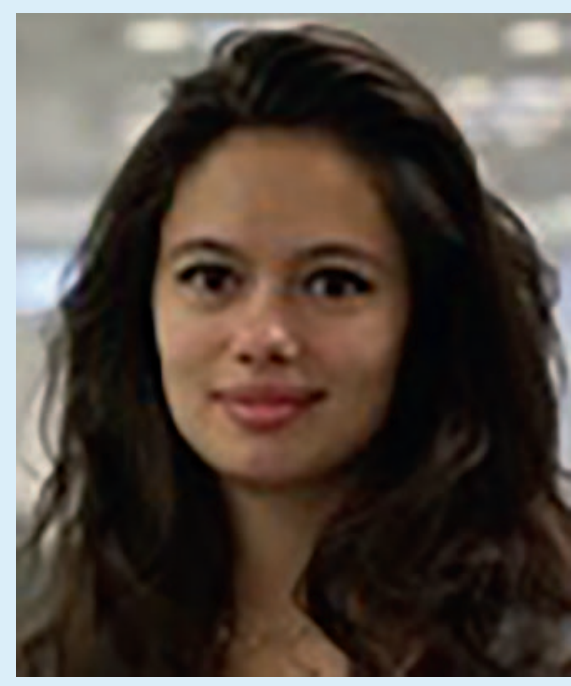

Sophie V Morse

and safety of drug delivery to treat sensitive neurological diseases, such as neurodegenerative diseases and paediatric brain tumours.

Sophie Morse was awarded a Masters in Engineering from Imperial College London in 2015 and then pursued a PhD in the Noninvasive Surgery and Biopsy Laboratory at Imperial working on drug delivery to the

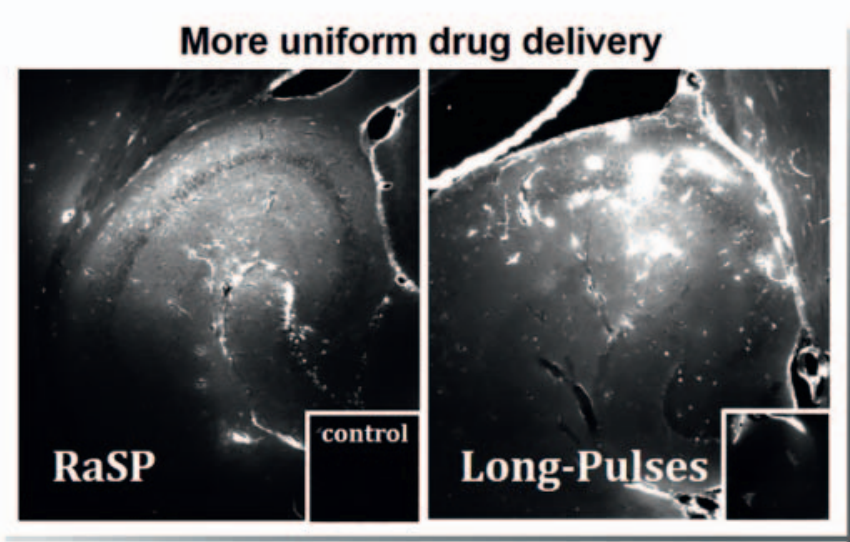

Less neurotoxic proteins in the brain

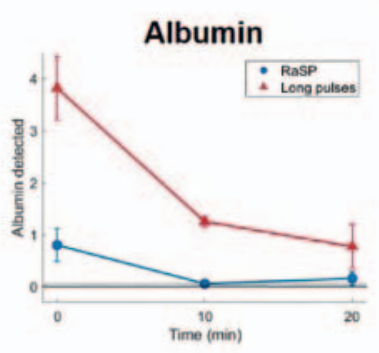

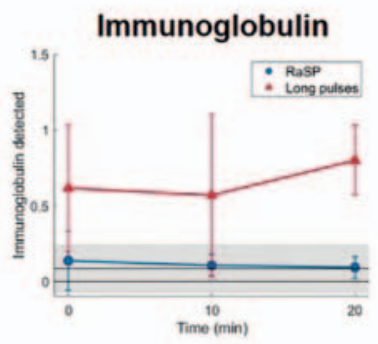

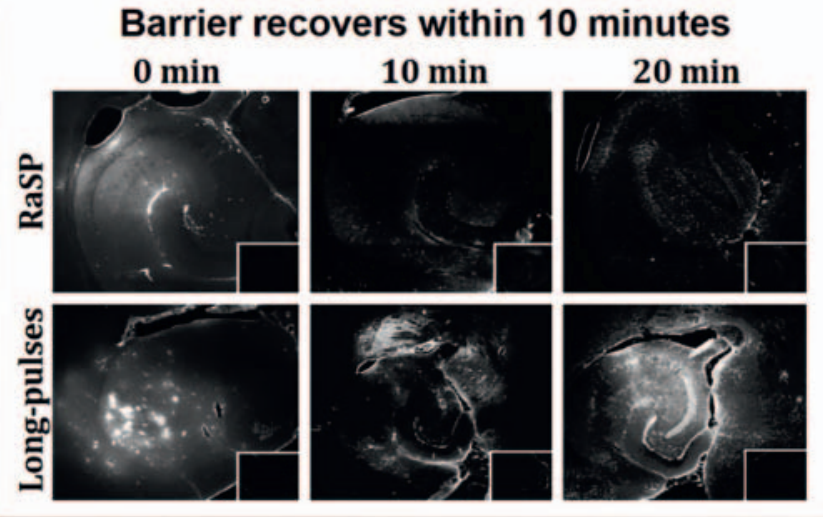

Less immune response

Uptake in Microglia

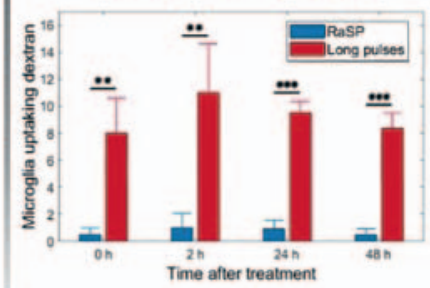

Uptake in Astrocytes

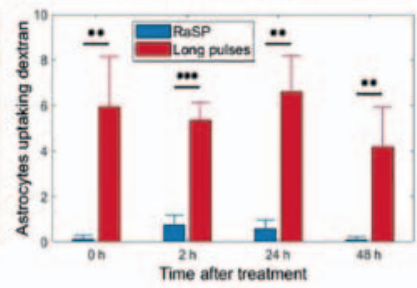


brain using focused ultrasound and microbubbles. She is currently an EPSRC doctoral prize research fellow in the Department of Bioengineering at Imperial College London. She has won numerous awards for her research including the Gold Medal in Engineering at the STEM for Britain event in the UK Parliament and the Image-guided therapy competition at the European Symposium on Ultrasound Contrast Imaging in Rotterdam. Her research interests include the non-invasive delivery of drugs and imaging agents to the brain and cellular stimulation using focused ultrasound.

\section{Joint first prize for the best}

\section{clinical presentation - \\ Rares Craciun, Romania}

\section{Ultrasound Guided Microwave Abla- tion and Transarterial Chemoembo- lization for Unresectable Solitary- Nodule Hepatocellular Carcinoma - a Head-to-Head Survival Compari- son}

Craciun Rares ${ }^{\mathbf{1 , 2}}$, Mocan Tudor ${ }^{1,2}$, Horhat Adelina $^{1,2}$, Teglas Cristian ${ }^{1}$, Nenu Iuliana ${ }^{1,2}$, Comsa Mihai ${ }^{1}$, Sparchez Zeno ${ }^{1,2}$

1 "Prof. Dr. O. Fodor" Regional Institute of Gastroenterology and Hepatology, Cluj-Napoca

2 "Iuliu Hațieganu" University of Medicine and Pharmacy, Cluj-Napoca

\section{ABSTRACT}

\section{Introduction}

To compare the overall survival (OS) of patients treated with ultrasound guided microwave ablation (MWA) and transarterial chemoembolization (TACE) for a solitary hepatocellular carcinoma (HCC) nodule.

\section{Methods}

A consecutive series of patients with a solitary, unresectable, HCC nodule under $5 \mathrm{~cm}$ in its largest diameter was prospectively enrolled from November 2015 to December 2019. The patients received either MWA or TACE. Treatment selection was decided by the institutional Tumor Board according to the most recent guidelines, considering tumor characteristics, underlying liver

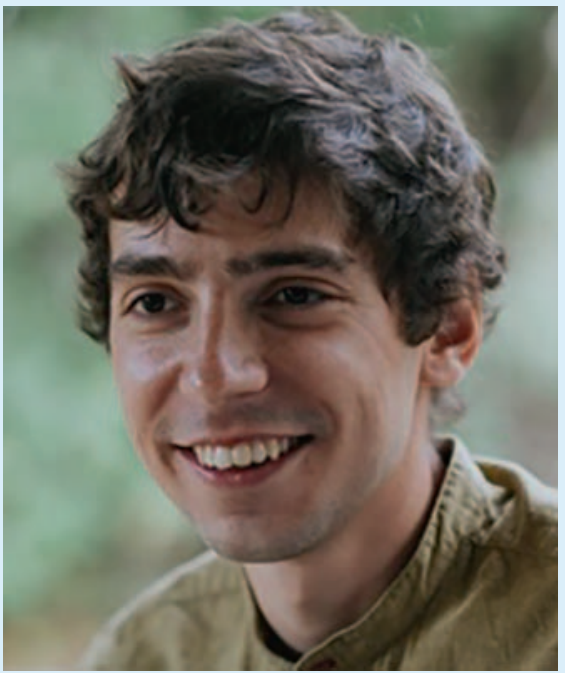

Craciun Rares

function and patient preference. OS was compared using the log-rank test.

\section{Results}

A total of 107 patients were enrolled, of which $n=77$ (71.9\%) were treated with MWA and $n=30$ (28.1\%) were treated with TACE. Patients in the TACE group had larger nodules ( $34 \pm 9$ vs. $23 \pm 8 \mathrm{~mm}, \mathrm{p}<0.001$ ). There were no other significant differences between groups with regards to patient characteristics. Complete response rate was higher in the MWA group ( $92.4 \%$ vs. $70 \%, p<0.001)$. OS was significantly higher in the MWA group: $52 \pm 3$ months vs. $24 \pm 1$ months for the TACE group (log-rank 10.25, $\mathrm{p}<0.001$ ). Subgroup analysis was performed for patients with tumor size exceeding $3 \mathrm{~cm}$. The difference in OS between groups persisted: patients who received MWA $(n=11)$ had an OS of $47 \pm$ 5 months vs. $22 \pm 2$ months for TACE (logrank 4.41, $\mathrm{p}=0.03$ ).

\section{Conclusion}

Ultrasound guided MWA provided a better outcome for patients with solitary unresectable HCC nodules, when compared to TACE. Whenever possible, prioritization of MWA appears to be desirable.

Born and raised in Transylvania, I am a GI and Hepatology fellow in training in my fourth year of practice. After receiving an MD degree at the "Iuliu Hațieganu" University of Medicine and Pharmacy, I have spent most of my professional formation within the "Prof. Dr. O. Fodor" Regional Institute of Gastroenterology and Hepatology in Cluj-Napoca, one of the largest tertiarycare facilities in Romania. In the past six years, my clinical focus was on hepatology, split between liver tumors and portal hypertension. Currently, I am a Ph.D. candidate, the research activity closely matching my clinical interest. To this point, I have coauthored over ten publications, mostly on interventional therapies in hepatocellular carcinoma and liver metastases, while being actively involved in the local and European scientific community.

\section{Review of the session for the EFSUMB Young Investigators Award 2021 by participant Sofie Bech Anderson}

On Saturday, May 29, the 2021 EFSUMB Young Investigators Award session was launched. Initially, the session was supposed to be held at EUROSON 2021 in Timisoara, Romania, but the congress was cancelled due to the status of the pandemic. Despite this, the nominated young investigators, including myself, got the chance to present and win the award, which included the title and a money prize. We were all invited to participate in an online award session: a format we are all quite familiar with at this point.

At first, EFSUMB planned the presentations to be prerecorded. However, they decided a few weeks before the session to make it live instead using Zoom. There is no doubt that this made the session more vivid and exciting. However, it also brought along the challenges of online meetings, including young children at home, unmuted computers or phones, and technical issues when sharing the presentations. Luckily, I think the last year and a half has taught us all a great deal of patience and understanding for each other's situations, working and presenting our results from home.

The seven young investigators presented a broad range of topics. In the more technically oriented category, three pre-clinical studies all dealt with microbubbles. The talks in this category illustrated how the 


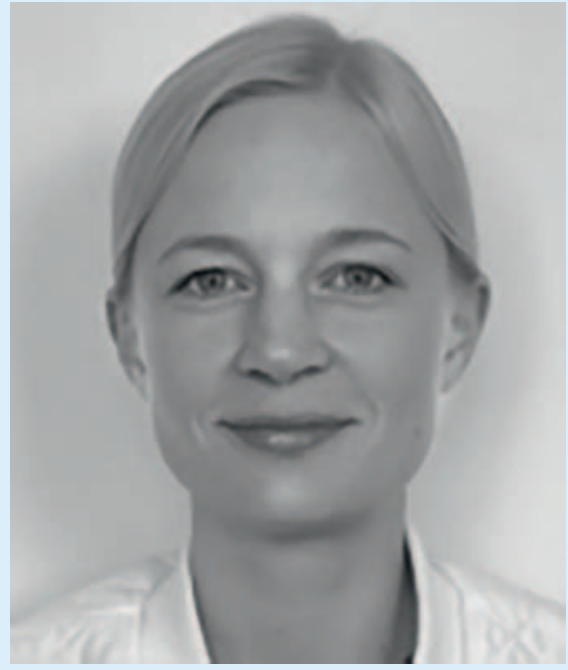

Sofie Bech Anderson

use of the tiny gaseous bubbles has expanded from being purely diagnostic to being both diagnostic and therapeutic. I gave a talk about how microbubbles make it possible to map the microvasculature at levels well below the diffraction limit of conventional ultrasound. This technique, called ultrasound super-resolution imaging, can potentially help us improve diagnostics and monitor diseases such as cancer and diabetes, where microvascular disruption plays a central role. Kirby Lattwein from the Netherlands showed how microbubbles can dissolve the devastating biofilm made by pathogenic bacteria: a technique called
Sonobactericide. Her results indicated that this treatment strategy has the potential to help thousands of persons affected by biofilm infections in the future. The winner of the technical part of the session, Sophie Morse from the UK, led us through her research on improved drug delivery to the brain with rapid short-pulses of focused ultrasound and microbubbles. She illustrated how instead of using long pulses of ultrasound, the rapid short-pulses improved the technique by, e. g., delivering drugs more uniformly to the tissue, closing the blood-brain barrier faster, and letting fewer neurotoxic proteins into the brain. This technique can improve drug delivery to the brain in the treatment of various neurological disorders.

In the clinically oriented talks, research in liver cancer diagnostics and treatment prevailed. The winner of this session, Rares Craciun from Romania, showed how ultrasound-guided microwave ablation provided a better overall survival for persons with solitary unresectable hepatocellular carcinoma nodules compared with transarterial chemoembolization, indicating that microwave ablation should be used for this group of patients whenever possible. Teodora Komitova from Bulgaria presented research showing that ablation can benefit persons with hepatocellular carcinoma at stage BCLC-B (Barcelona clinic liver cancer (BCLC) staging) and cirrhosis stage Child-Pugh B. Marco Arru from Italy also investigated the liver, but with a diagnostic aim. He had studied the contrast-enhanced ultrasound Liver Imaging Reporting And Data System (LI-RADS) as a diagnostic tool to better differentiate hepatocellular carcinoma and intrahepatic cholangiocarcinoma. Last but not least, and with a very different topic than the other clinical presentations, Tasias Konstantinos from Greece presented his research on the prevalence of agenesis of ductus venosus (ADV) in an unselected population, the types of drainage in ADV and the associated structural or chromosomal abnormalities as well as the postnatal outcome in these cases.

After all the presentations were done, the judges went into a break-out room to decide on the winners. It was a peculiar experience, waiting alone behind the screen, but the moderators made a great effort to make us all entertained and fill the silence.

After the winners had been announced, kind congratulations to all the participants ticked silently into the chat. We left the session to sit in each of our respective countries. I sat in my empty office at the empty university on a sunny summer day. It was a quiet way to end the experience. I really hope that next year's participants get to meet and present in person. 